\title{
1 Rational Curiosity and Information-Seeking in the COVID-19 Pandemic
}

2 Yaniv Abir ${ }^{1 *}$, Caroline B. Marvin ${ }^{1}$, Camilla van Geen ${ }^{2}$, Maya Leshkowitz ${ }^{3}$, Ran R. Hassin ${ }^{4 \dagger}$,

3 Daphna Shohamy ${ }^{1,2,5 \dagger}$

$4 \quad{ }^{1}$ Department of Psychology, Columbia University, New York, NY, United States.

$5{ }^{2}$ Zuckerman Mind Brain Behavior Institute, Columbia University, New York, NY, United States.

$6 \quad{ }^{3}$ Department of Cognitive Sciences, The Hebrew University of Jerusalem, Jerusalem, Israel.

$7 \quad{ }^{4}$ Department of Psychology and The Federmann Center for the Study of Rationality, The Hebrew

8 University of Jerusalem, Jerusalem, Israel.

$9{ }^{5}$ Kavli Institute for Brain Science, Columbia University, New York, NY, United States.

10 *Corresponding author: yaniv.abir@columbia.edu

11 These authors contributed equally to this work.

\section{Abstract}

13 Curiosity is a powerful determinant of behavior. The past decade has seen a surge of scientific

14 research on curiosity, an endeavor recently imbibed with urgency by the WHO, which set

15 managing information-seeking as a public health goal during pandemics. And yet, a fundamental

16 aspect of curiosity has remained unresolved: its relationship to utility. Is curiosity a drive towards

17 information simply for the sake of obtaining that information, or is it a rational drive towards

18 optimal learning? We leveraged people's curiosity about COVID-19 to study information-seeking

19 and learning in a large sample $(n=5376)$ during the spring of 2020. Our findings reveal that

20 curiosity is goal-rational in that it maximizes the personal utility of learning. Personal utility,

21 unlike normative economic utility, is contingent on a person's motivational state. On the basis of 
22 these findings, we explain information-seeking during the pandemic with a rational theoretical

23 framework for curiosity. 
24 Curiosity is the drive that determines how we consume information in a world where information

25 abounds ${ }^{1,2}$. Despite being a powerful determinant of behavior, curiosity remains poorly

26 understood, in part due to its paradoxical relation to utility: on the one hand, curiosity is often

27 seen as the drive for knowledge for its own sake, regardless of its utility, or even in spite of its

28 disutility ${ }^{1-5}$. On the other, curiosity is the engine behind learning and development, hence clearly

29 useful for the organism ${ }^{6-10}$. While there is support for each of these perspectives separately,

30 surprisingly little is known about whether and how curiosity is shaped by utility.

31 The prevalent conviction that curiosity is the drive for useless information is grounded not only in 32 a long philosophical tradition ${ }^{1,2}$, but, more recently, also in the failure of economic models of

33 utility to explain the extent of human curiosity, and why humans commonly overpay for

34 information relative to an economically defined norm ${ }^{1,11}$. Researchers have thus been motivated

35 to construct tasks in which only useless information is offered to participants ${ }^{3,11,12}$. This approach

36 revealed that when utility cannot guide curiosity, the amount of information on offer determines

37 information-seeking choices.

38 In contrast, the clear adaptive value of curiosity as an enhancer of memory and learning $9,10,13,14$

39 and as a predictor of academic success ${ }^{15}$ inspired the formulation of several normative theories of

40 curiosity. These recent theoretical advances describe curiosity as a drive to maximize both

41 information and utility ${ }^{6-8,16}$. However, such theories acknowledge that deriving the exact utility of

42 an answer to a question is generally an intractable problem ${ }^{16,17}$. They postulate that human

43 curiosity must instead approximate this computational goal. In what way human curiosity

44 approximates utility maximization, however, remains unknown. 
45 The 2020 coronavirus pandemic (COVID-19) created a unique opportunity to examine the

46 relationship between utility and curiosity. Suddenly, many people were curious about the virus

47 and its epidemiology, a topic they were largely ignorant about before, but which became

48 motivationally relevant as well as intellectually interesting. Furthermore, differences in age,

49 geography and circumstance resulted in considerable individual differences in the personal

50 relevance of information regarding COVID-19. Thus, a rare opportunity emerged to study

51 ecologically valid information-seeking, while measuring naturally occurring variations in utility

52 and motivation.

53 Additionally, the WHO has designated the seeking and sharing of information a key area for

54 intervention during the COVID-19 pandemic $^{18}$. However, an understanding of information-

55 seeking under such conditions is lacking, with conflicting views regarding the ability of humans

56 to rationally sift through the informational deluge ${ }^{19-22}$. Examining the relationship between

57 curiosity and utility addresses this pertinent gap in scientific evidence.

58 We therefore leveraged the conditions created by the pandemic to investigate curiosity and

59 learning. Based on our findings, we propose a rational framework for curiosity. This framework

60 places utility as a central driver of curiosity and learning. It suggests that curiosity is shaped by a

61 cost-benefit analysis between the expected utility of obtaining information and the cost of seeking

62 that information. Critically, according to this view, individuals derive their personal expected

63 utility both from the content of information as well as from their motivational state. We show that

64 curiosity covaries with personal utility in a manner largely consistent with normative theory, but

65 also detail how it diverges from it. 
66 To investigate the role of utility in curiosity we used Amazon Mechanical Turk to gather data

67 from 5,376 participants across the United States, sampling twice a week between March 11th and

68 May 7th, 2020. We assessed how participants' choices to seek information were affected by its

69 usefulness and its relatedness to COVID-19, as well as by participants' expectations and concerns

70 regarding the COVID-19 pandemic.

71 We measured participants' willingness to wait for information as a behavioral marker of curiosity

72 (Fig. 1, a\&b). Participants (ages 18-89) read a series of questions relating either to the

73 coronavirus pandemic or to general trivia and everyday tips (Table S2). For each question,

74 participants had to indicate whether they already knew the answer, would like to skip it, or were

75 willing to wait a specified number of seconds to receive the answer. Importantly, participants

76 knew that the entire duration of the experiment was independent of their choices and therefore

77 were encouraged to use their own curiosity to decide whether to wait. The proportion of 'wait'

78 versus 'skip' responses at variable waiting durations serves as our main index of curiosity ${ }^{13}$. One

79 week later, we asked participants to recall the answer to each question they had waited for. Their

80 memory performance complements our measure of curiosity, enabling us to track how curiosity

81 drives behavior from information-seeking to learning.

82 To assess the personal utility of the information, three additional measures were obtained. After

83 completing the waiting task, each participant was presented with a subset of new questions and

84 rated each question on the expected usefulness of its answer, both for themselves and for others.

85 Additionally, at the end of the experiment, participants completed a questionnaire assessing how

86 concerned they were about the developing COVID-19 pandemic and the changes it could cause in

87 their lives (henceforth "COVID-19 concern"). Prior to this, participants answered a series of 
88 questions regarding their non-specific anxiety and affective state (henceforth "non-specific

89 anxiety"), which allowed us to compare the effect of a domain-specific concern on curiosity with

90 the effect of anxiety in general. Our use of these two measures is predicated on the view that

91 affective states are motivational states $^{23,24}$.

92 We hypothesized that curiosity should be determined by the content of each question, which we

93 operationalize as each question's relatedness to COVID-19, and its average usefulness judgment.

94 However, we reasoned that just as a food reward does not have the same utility for a hungry

95 subject and a satiated one, the same question should elicit varying expectations of utility from

96 participants in different motivational states ${ }^{25,26}$. Thus, differences in participants' motivational

97 states should result in different mappings between question content (COVID-19-related or not)

98 and utility. We define personal utility as this interaction between motivation and content and

99 hypothesize that it predicts curiosity-driven behavior.

\section{$100 \quad$ Results}

101 Cost-benefit rationality

102 Our findings reveal that, in accordance with a rational framework, curiosity is cost-benefit

103 rational (Fig. 1, c\&d): participants are more likely to wait for questions judged as useful, whether

104 these are COVID-19-related or general questions $b=0.66,95 \%$ posterior interval $(\mathrm{PI})=[0.53,0.79]$. 
105 As expected from a cost-benefit analysis, willingness to wait also diminishes for longer wait

106 durations $b=-0.07,95 \% \mathrm{PI}=[-0.07,-0.06]$

a

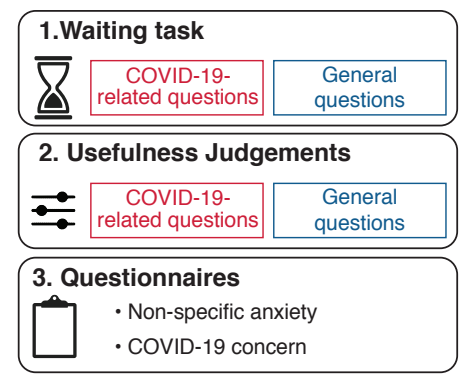

4. Answer recall after one week

进焦 $\begin{gathered}\text { COVID-19- } \\ \text { related questions }\end{gathered} \begin{gathered}\text { General } \\ \text { questions }\end{gathered}$

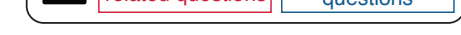

b COVID-19-related

What is the source

of coronavirus in

humans?

WAIT Xs SKIP
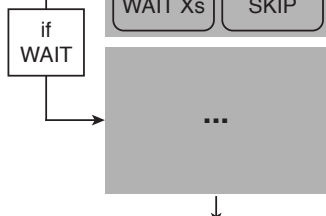

$\downarrow$

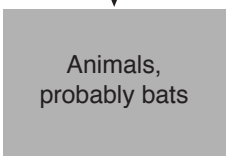

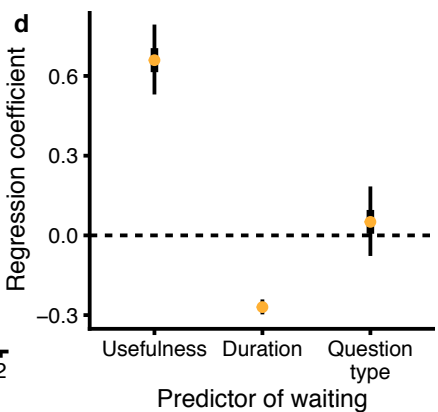

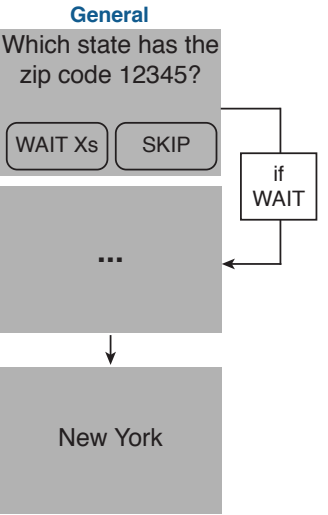

c

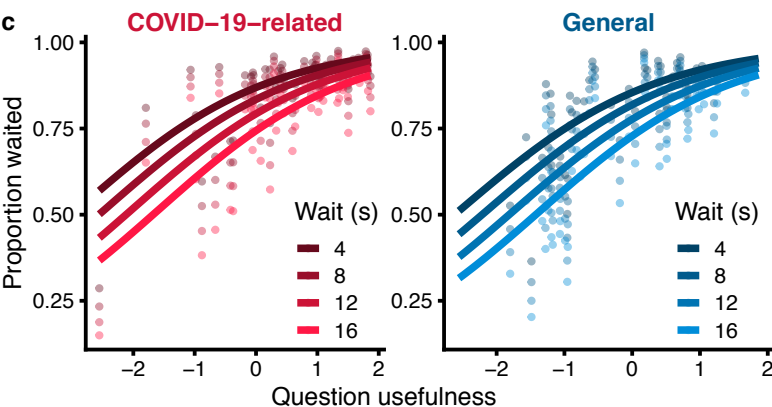

Figure 1. Curiosity is cost-benefit rational. (a) General outline of the four stages of the experiment. (b)

109 On each trial of the waiting task, participants were presented with a question. If they decided to wait a

110 specified duration, they were presented with the answer. They were then asked to rate their satisfaction

111 with the answer and, one week later, to report their memory (not pictured). (c) Participants were more

112 likely to wait for questions judged as more useful, and less likely to choose to wait long durations. These

113 effects hold for both general and COVID-19 related questions. Lines denote predictions from a multilevel

114 logistic regression model; dots mark marginal proportions for questions. (d) Coefficients from the

115 regression model with $50 \%$ and $95 \%$ PIs.

116 Goal-rationality

117 Before addressing the role of motivational states in driving curiosity, we first verified that our two

118 affective measures (COVID-19 concern and non-specific anxiety) were related to relevant real-

119 world experiences. As expected, we found that both measures vary with events such as job loss, 
120 income reduction, self-isolation, social distancing behavior in the state, and the timeline of the 121 virus spread in the U.S. (see Methods).

122 Having verified the validity of our affective measures, we then tested the hypothesis that curiosity 123 is sensitive to participants' motivation, and as such is goal-rational. First, we focused on the effect 124 of COVID-19 concern on curiosity towards different types of questions. We found that people 125 reporting higher COVID-19 concern were more curious about COVID-19-related questions 126 relative to general questions $b=0.11,95 \% \mathrm{PI}=[0.08,0.14]$ (Fig. 2a). This is in agreement with the 127 rational framework, which predicts that a change in domain-specific motivation should impact 128 curiosity towards information in that domain. The rational framework further posits that domain129 specific motivation operates on curiosity by increasing the expected utility of questions in the 130 relevant domain. Indeed, we find that higher COVID-19 concern is associated with higher 131 usefulness judgments of COVID-19-related questions relative to general questions $b=0.10,95 \%$ $132 \mathrm{PI}=[0.07,0.12]$ (Fig. 2c). Furthermore, a mediation analysis revealed a significant indirect effect 133 of the interaction of COVID-19 concern and question type (COVID-19-related/general) on 134 waiting choices, via usefulness judgments $b=0.02,95 \% \mathrm{PI}=[0.002,0.04], 22.27 \%$ of the effect 135 mediated, 95\% PI=[1.71\%, 49.86\%] (Fig. 2e).

136 A truly normative account would predict that COVID-19 concern would only engender the 137 changes in curiosity for COVID-19-related content, as described above. By contrast, mindset 138 theories $^{27,28}$, as well as computational theories of motivation ${ }^{25,26}$, predict that as COVID-19 139 concern changes the utility of COVID-19-related information, it should also change the average 140 utility of information pursuit in general, leading to generalized effects on curiosity. Our results 141 are consistent with the latter interpretation: we find that in addition to its specific effects, COVID- 
14219 concern has a general positive effect on curiosity and utility, as it is associated with increased

143 waiting also for general questions $b=0.28,95 \% \mathrm{PI}=[0.20,0.37]$ (Fig. 2a), and also with a general

144 rise in usefulness judgments $b=0.51,95 \% \mathrm{PI}=[0.47,0.55]$ (Fig. 2c). A mediation analysis

145 confirmed a significant indirect effect of COVID-19 concern on waiting via usefulness judgments

$146 \mathrm{~b}=0.14,95 \% \mathrm{PI}=[0.01,0.25], 69.27 \%$ of the effect mediated $95 \% \mathrm{PI}=[5.38 \%, 151.16 \%]$ (Fig. 2f).

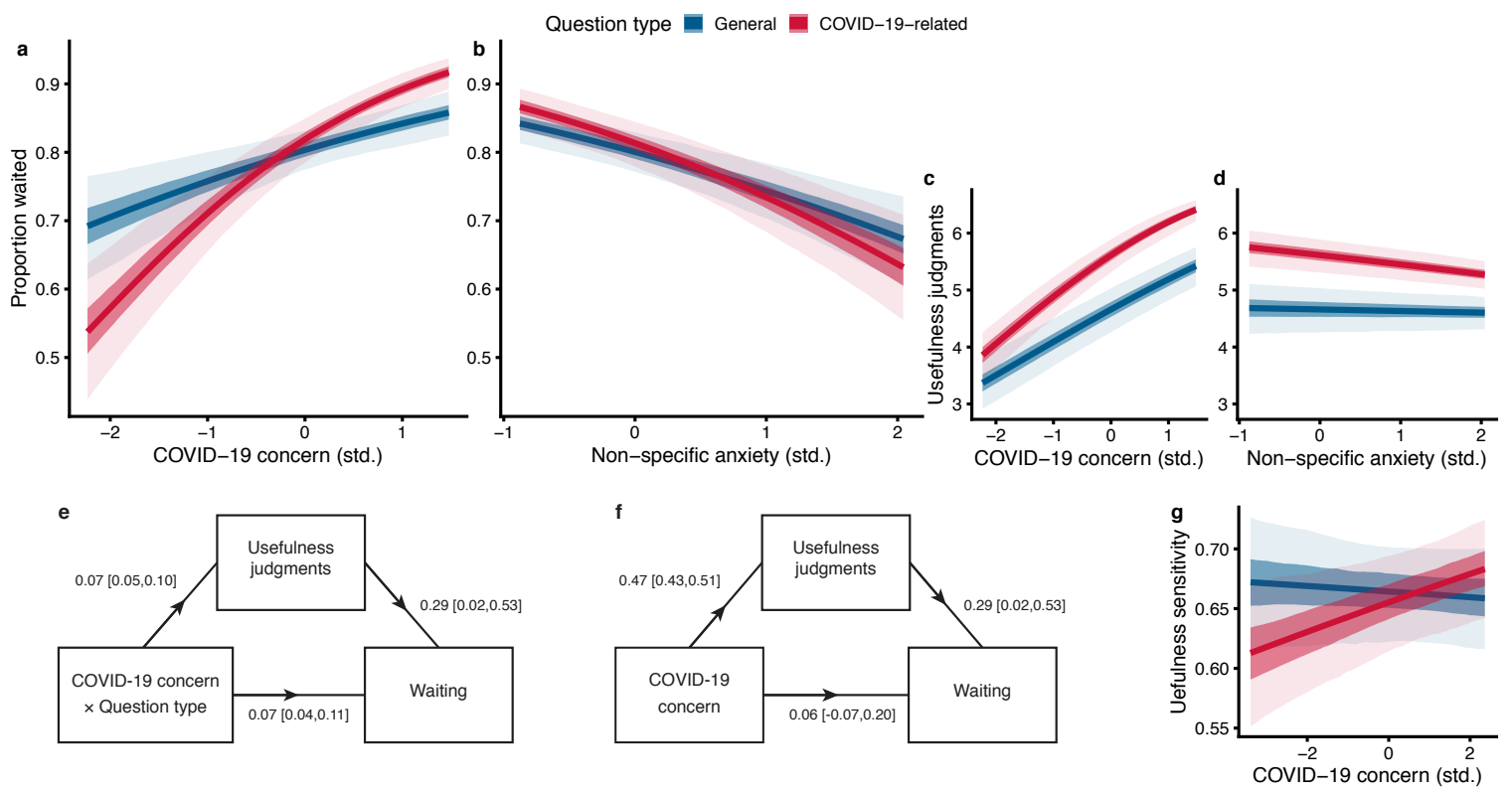

Figure 2. Motivational states modulate curiosity. (a) Higher COVID-19 concern is associated with more

149 waiting for COVID-19 related questions, and also, but to a lesser degree, for general questions. (b) Non-

150 specific anxiety negatively predicts waiting for all questions. (c) Participants reporting higher COVID-19 concern judge questions as more useful, especially COVID-19-related questions. (d) Participants reporting

152 higher levels of non-specific anxiety tend to judge questions as less useful, especially COVID-19-related 153 questions. (e) Consistent with a rational framework for curiosity, according to which the specific effect of

154 COVID-19 concern on curiosity should be mediated via personal utility, we find a significant indirect

155 effect of the interaction between COVID-19 concern and question type on waiting, mediated by judged

156 usefulness. 95\% PIs given in brackets. (f) We also find a significant indirect main effect of COVID-19

157 concern, via judged usefulness, on waiting, consistent with the motivational account of curiosity in which

158 the generalized effect of COVID-19 concern on curiosity is mediated via personal utility. (g) Participants

159 who report more COVID-19 concern have a tendency to be more sensitive to the usefulness of COVID-19- 
160 related questions when deciding whether to wait for their answers $b=0.05,95 \% \mathrm{PI}=[0.006,0.10]$. COVID-

16119 concern, however, is not related to usefulness sensitivity for general questions $b=-0.01,95 \% \mathrm{PI}=[-$

$1620.06,0.04]$. Lines denote mean posterior prediction; dark shaded areas mark 50\% PIs, and light areas 95\%

163 PIs.

164 A general effect of COVID-19 concern on curiosity could imply that people concerned with

165 COVID-19 are just less discerning in their information-seeking. The data do not support such an

166 account - COVID-19 concern is not associated with a reduction in sensitivity to question

167 usefulness when making waiting choices $\mathrm{b}=0.02,95 \% \mathrm{PI}=[-0.01,0.06]$. One's personal

168 motivation does seem to affect the extent to which one is sensitive to usefulness when seeking

169 information in different content domains. We find that among people with higher COVID-19

170 concern, choices regarding COVID-19-related questions are more sensitive to usefulness, relative

171 to choices regarding general questions $b=0.03,95 \% \mathrm{PI}=[-3.45 \mathrm{e}-04,0.06]$ (Fig. $2 \mathrm{~g}$ ).

172 Importantly, the effects of COVID-19 concern on curiosity do not appear to be due to general

173 anxiety. While COVID-19 concern and non-specific anxiety are moderately correlated, we

174 observe that they predict curiosity in starkly different ways. Participants reporting high levels of

175 non-specific anxiety were less likely to wait for answers of any sort $b=-0.28,95 \%$ PI $=[-0.37$,-

$1760.20]$ (Fig. 2b). This diminished curiosity parallels previous findings of diminished pursuit of

177 reward among people with depression ${ }^{24,29,30}$.

178 Finally, we tested whether this pattern of results, supporting goal-rationality, generalize beyond

179 the waiting task. See supplementary Information and Fig. S2 for a successful conceptual

180 replication using a self-report measure of curiosity. 

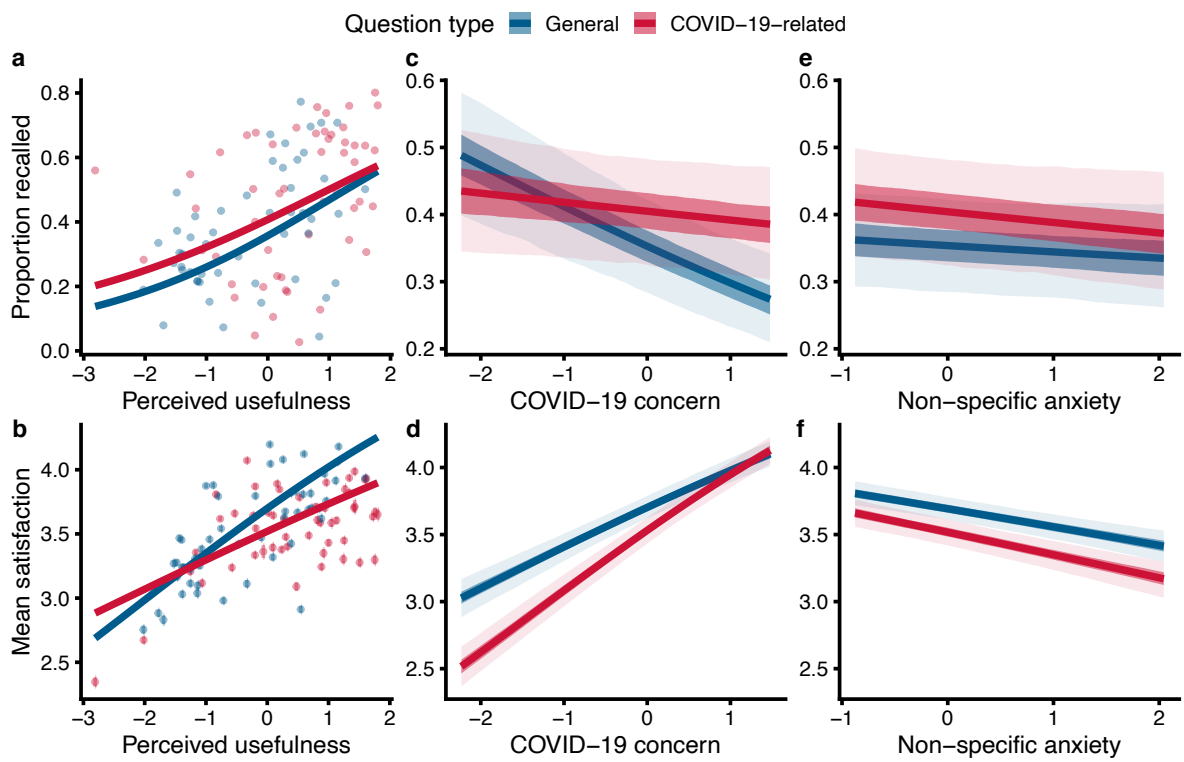

182 Figure 3. Motivation modulates subsequent memory for the answers to questions and subsequent satisfaction with the answers. (a) Judged usefulness is associated with better memory for the answer, assessed with a memory test one week after the waiting task. (b) Judged question usefulness is also positively correlated with self-reported satisfaction with answers. (c) COVID-19 concern is associated with

186 poorer memory for general information, while information related to COVID-19 is spared. (d) Greater

187 COVID-19 concern is associated with more self-reported satisfaction, especially for COVID-19 related questions. (e) Non-specific anxiety is not significantly associated with memory $b=-0.04,95 \% \mathrm{PI}=[-$ $0.08,0.003]$. (f) Non-specific anxiety is associated with a reduction in satisfaction for both question types $\mathrm{b}=-0.20,95 \% \mathrm{PI}=[-0.25,-0.15]$. Lines denote mean posterior prediction from multilevel regression models;

191 dots mark marginal means for questions; dark shaded areas mark 50\% PIs, and light areas 95\% PIs.

192 Response to answers and subsequent learning

193 A role for utility in driving curiosity is rational only inasmuch as this relationship is manifested in

194 subsequent learning. Indeed, personal utility is a central predictor not only of curiosity, but also of 195 participants' long-term memory for the answers. We measured memory after one week and found 196 that participants better remembered answers to questions that were judged as highly useful $197 \mathrm{~b}=0.42,95 \% \mathrm{PI}=[0.21,0.64]$ (Fig. 3a). Crucially, higher COVID-19 concern predicted poorer 
198 memory for general answers, but spared COVID-19-related answers (interaction $b=0.06,95 \%$

$199 \mathrm{PI}=[0.03,0.09]$; Fig. 3c), a finding compatible with an adaptive forgetting account ${ }^{31,32}$.

200 Similarly, we found that personal utility also affected participants' subjective reports of

201 satisfaction, measured after each answer was shown. Like curiosity, answer satisfaction increases

202 with question usefulness judgments $b=0.53,95 \% \mathrm{PI}=[0.42,0.65]$ (Fig. 3b). We further found that

203 people high in COVID-19 concern were more satisfied with all answers $b=0.43,95 \%$

$204 \mathrm{PI}=[0.38,0.48]$, but especially COVID-19-related answers (interaction $b=0.08,95 \%$

$205 \quad \mathrm{PI}=[0.06,0.11]$; Fig. 3d).

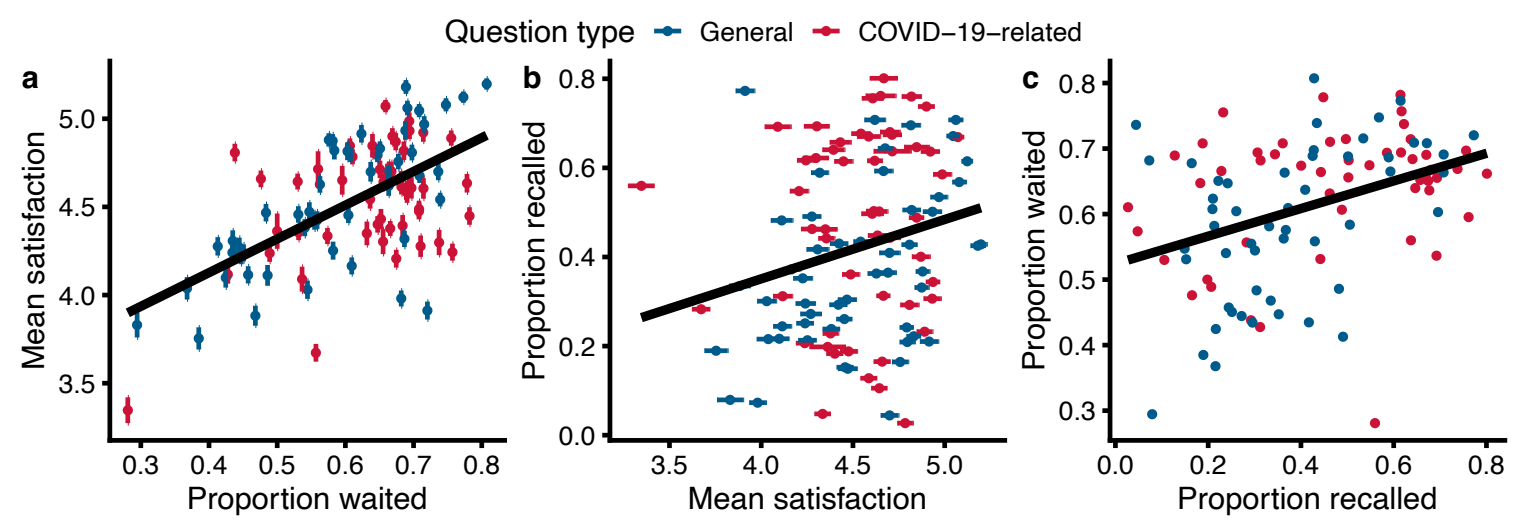

207 Figure. 4. Participants tend to seek information that on average would make them more satisfied,

208 and that they are more likely to remember. (a) Questions for which a high proportion of participants

209 chose to wait tend to have satisfying answers, and vice versa $r=0.60, p<0.001$. (b) Satisfaction with

210 answers is correlated with probability of recalling them $r=0.23, p=0.02$. (c) Proportion of participants

211 waiting for a question is correlated with the proportion of participants recalling the answer to said question

$212 \mathrm{r}=0.39, \mathrm{p}<0.001$. Error bars span two standard errors. Linear regression lines are plotted for visualization

213 purposes.

214 In general, we find that people tend to seek answers that they would subsequently be satisfied

215 with and remember well (correlation between waiting and satisfaction $\mathrm{r}=0.60, \mathrm{p}<0.001$; between 
216 waiting and memory $r=0.39, \mathrm{p}<0.001$; Fig. 4). Taken together, these findings indicate that

217 personal utility influences not only information seeking, but also subsequent processing of the

218 information, with long-term consequences for learning.

\section{Discussion}

220 Together, these findings support the hypothesis that curiosity is the pursuit of useful

221 information ${ }^{7,8}$. Moreover, these results uncover the psychological mechanisms of this process. We

222 find that humans behave as if weighing the cost of information-seeking against the personal

223 utility of information, which is derived from the content of a question according to the

224 motivational state of each individual.

225 Deriving the exact personal utility of information based on one's motivation and goals is

226 intractable computationally, and so must be approximated by any intelligent system ${ }^{16,17}$. We

227 observe that the human cognitive system is largely rational in its approximation of personal

228 utility. Thus, specific affective states, or motivations, bring about domain-specific changes in

229 personal utility and curiosity - the hallmark of goal-rational behavior. However, these

230 motivational states are also associated with general changes in curiosity towards all content

231 domains, through the influence of motivation on the average expected utility of information. The

232 use of average expected utility in decisions is a useful simplification from a computational

233 perspective, but constitutes a deviation from purely normative behavior ${ }^{25,26}$.

234 Our focus here was on the specific and generalized effect of COVID-19 concern. However, we

235 also observed a generalized effect of non-specific anxiety on curiosity, whereby more anxious

236 people tended to seek less information. This dissociation between COVID-19 concern and non- 
237 specific anxiety was important to establish our main conclusions. However, this finding also has

238 clinical significance in its own right, as some previous work found support for diminished

239 curiosity $^{30}$, while other researchers postulated that anxiety might increase information-seeking, as

240 it is associated with high intolerance for uncertainty ${ }^{33-35}$. Furthermore, the negative correlation

241 between non-specific anxiety and information-seeking in our data mirrors established findings

242 regarding diminished reward-seeking in anxiety and depression ${ }^{24,29}$.

243 This rational framework of curiosity and the findings that support it strive to explain curiosity

244 using the same computational and algorithmic principles derived from studying reward-based

245 behavior $^{4,14,36}$. Recently, curiosity has been implicated as a necessary core computation for natural

246 and artificial intelligence ${ }^{37,38}$, with information and reward postulated to be the basic fungible

247 currencies of cognition ${ }^{6,16,36}$. Explaining ecological curiosity in cost-benefit terms is a step

248 towards understanding the economy of utility and information in the brain.

249 Finally, our findings address key open questions about how people seek information during the

250 COVID-19 pandemic $^{18}$. Whether people seek information rationally, and can thus be trusted to

251 consume useful information, is the subject of active debate in public health and political science

252 research ${ }^{\text {e.g. }}$ 19-22. Our findings suggest that humans are efficient information seekers, and that the

253 measurement of affective and motivational factors as modulators of personal utility is an

254 important tool in understanding individual information-seeking behavior during an epidemic, or

255 other events of interest for public policy.

\section{Methods}

257 Data collection 
258 Participants were recruited through Amazon Mechanical Turk, with data collection occurring

259 twice weekly, between March 11th and May 7th 2020. A week after their participation in the first

260 session, participants received an email inviting them to participate in session 2 of the study.

261 Overall, $71.48 \%$ of eligible participants returned for session 2.

262 All subjects provided informed consent; all protocols were approved by the Columbia University

263 Institutional Review Board. Detailed data collection descriptions are available in Supplementary

264 Information.

$265 \underline{\text { Stimuli }}$

266 A set of short questions and answers was used as stimuli in this experiment. 52 COVID-19-

267 related questions were sourced from materials published by the World Health Organization, US

268 Center for Disease Control and Prevention, or the New York Times. Half of these were deemed

269 useful by the authors of the study and half non-useful. 52 general questions comprised the second

270 type of questions - half of these were trivia questions drawn from previous studies ${ }^{14}$, and half

271 were useful household tips sourced from lists of tips on the internet. See Table S2 for list of

272 questions.

273 Task Design

274 In the first experimental session, participants first completed the waiting task, with a block

275 comprising COVID-19-related questions and another comprising general questions, in

276 counterbalanced order. They then rated a held-out set of questions on the expected usefulness of

277 answers, both for themselves and for others. At the end of the session, participants completed a

278 questionnaire regarding their general affective state, followed by a questionnaire probing their

279 affective concerns regarding COVID-19. This questionnaire included items regarding anxiety 
280 about the medical, economic and social circumstances, and perceptions of severity and risk (see

281 Table S3 for list of questions). During the second session of the experiment, held 7-8 days after

282 the first, participants completed the answer recall task.

283 On each trial of the waiting task, participants were presented with a question, and three choice

284 buttons. If they knew the answer to the question, they were instructed to press 'know'. Otherwise,

285 they could choose to wait a specified duration for the answer by pressing 'wait Xs', or else

286 choose to press the 'skip' button, which terminated the trial. Durations were assigned in random

287 order from the set $\{4 \mathrm{~s}, 8 \mathrm{~s}, 12 \mathrm{~s}, 16 \mathrm{~s}\}$. An ellipsis was presented during the waiting period, followed

288 by the answer. Participants were asked to rate their satisfaction with each answer on a 1-5 Likert

289 scale. The duration of each waiting task block was set to 180 s, regardless of participants' choices.

290 On each trial of the recall task, participants were presented with a question they had chosen to

291 wait for a week earlier. Participants indicated whether they remembered the answer to the

292 question. If so, they had to input the answer into a text box.

293 Complete timing parameters are given in the Supplementary Information, see Fig. S1 for 294 screenshots of the task.

$295 \underline{\text { Analysis }}$

296 Data was analyzed using the R statistical environment 3.6.0, and Stan probabilistic language

297 2.23.0. Julia 1.4.2 scripts were used to parse raw data and model outputs for greater

298 computational speed.

299 Exclusion criteria. Four participants reported technical difficulties in the presentation of 300 questions. Their data was excluded from analysis. Data from 358 participants (5.84\%) reporting 
301 less than perfect English language fluency, and 335 participants (5.46\%) who interacted with

302 other applications more than 5 times during the waiting or rating tasks were further excluded

303 from analysis. Following previous studies with the waiting task ${ }^{14}$, we excluded data from

304 participants who failed to respond on more than $20 \%$ of trials $(n=4,0.07 \%)$, or whose mean

305 response time was more than 2 standard deviations (SD) lower than the group average ( $\mathrm{n}=58$,

306 0.95\%). Overall, data from 5376 participants was included in analyses (median age 36, range 18-

$307 \quad 89 ; 2818$ female).

308 We separately excluded data from the second session if participants had more than 5 application

309 interactions during the recall task $(n=176,3.27 \%)$, or inputted responses that were coded as non-

310 compliant with instructions (e.g. non-words, $n=52,0.97 \%$ ).

311 Scoring memory recall. Participants recollected answers were compared to the original answer

312 they had seen. Exact matches were scored as correct by an R program. A research assistant blind

313 to the research hypotheses and conditions scored the remaining responses as either incorrect, not

314 recalled (e.g. "I don’t remember"), partially correct, or correct. 311 responses $(0.90 \%)$ were

315 flagged as not compatible with instructions. Both partially correct and correct answers are

316 considered as successful recollection in all subsequent analysis, while incorrect and not recalled

317 are considered as recollection failures.

318 Validation of motivational state measures. Ratings for the all affective items in the experiment

319 were subjected to a Bayesian Principal Component Analysis ${ }^{39}$, which is robust to missingness in

320 the data. Five-fold cross validation revealed that three was the optimal number of components in

321 the data. The three components were rotated using the Quartimax method ${ }^{40}$, and each item was

322 assigned to the component on which it had the strongest loading. One group contained items 
323 related to COVID-19, comprising the COVID-19 concern measure, while another contained all

324 items measuring negative affect, which we used as the non-specific anxiety control. The third

325 component contained all positive affect items. We used the unweighted means of each variable

326 group to avoid overfitting. The relation between affective measures and curiosity are very similar

327 when using a naive grouping of items, according to the original questionnaire they came from.

328 To further establish the validity of our COVID-19 concern and non-specific anxiety measures, we

329 sought to relate them to participants' real-world circumstances. We found that ratings of COVID-

33019 concern were higher for participants who experienced job loss $t(1069.84)=12.49, \mathrm{p}<0.001$, a

331 reduction in income $\mathrm{t}(3790.22)=12.26, \mathrm{p}<0.001$, or self-isolation $\mathrm{t}(2129.50)=7.98, \mathrm{p}<0.001$,

332 relative to participants who did not experience these events (Fig. 5d). A similar pattern was

333 observed for non-specific anxiety (job loss: $\mathrm{t}(930.69)=15.42, \mathrm{p}<0.001$, income decrease:

$334 \mathrm{t}(3448.08)=13.94, \mathrm{p}<0.001$, self-isolation: $\mathrm{t}(2482.32)=5.42, \mathrm{p}<0.001$; all degrees of freedom are

335 given with the Welch correction for unequal variances).

336 We compared rates of COVID-19 concern and non-specific anxiety between states with different

337 levels of social distancing, and across timepoint from mid-March to early May 2020. These

338 effects were evaluated with a multilevel non-linear general additive model (GAM). We find a

339 significant non-linear component in the development of COVID-19 concern over time $\mathrm{SD}=0.76$,

$34095 \% \mathrm{PI}=[0.36,1.45]$, but not a linear increase $\mathrm{b}=1.15,95 \% \mathrm{PI}=[-0.23,2.42]$. As can be seen in

341 Fig. 5a, COVID-19 concern rose during the last two weeks of March, before plateauing and

342 gradually decreasing. A similar non-linear component is found for non-specific anxiety $\mathrm{SD}=0.53$,

$34395 \% \mathrm{PI}=[0.16,1.14]$. 


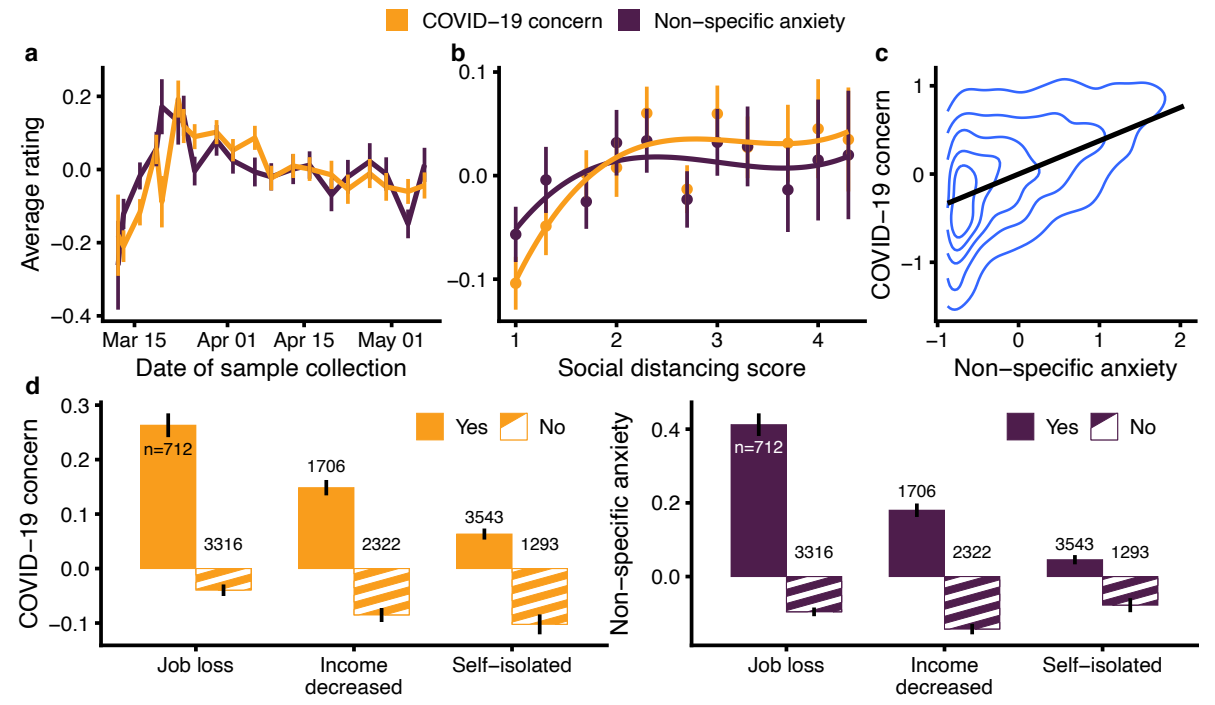

345 Figure 5. Motivational state metrics track with real-world circumstances. (a) Standardized ratings of COVID-19 concern increased sharply mid-March 2020, a similar pattern is observed

347 for non-specific anxiety. (b) COVID-19 concern is higher for people in states where social

348 distancing is practiced, as measured by Unacast via smartphone location data ${ }^{44}$. Lines are

349 predictions from a general additive model. (c) Non-specific anxiety and COVID-19 concern are

350 moderately correlated $r=0.44, p<0.001$; black line derived from linear regression. (d) COVID-19

351 concern levels (plotted on the left) are higher among participants who had lost their jobs, had seen

352 their income decrease, or went into self-isolation. The same was true for specific anxiety, plotted

353 on the right. The number of participants in each group is noted near each bar. All error bars span

354 two standard errors.

355 We observed a significant non-linear component in the change of COVID-19 concern with the

356 social distancing measure $\mathrm{SD}=0.11,95 \% \mathrm{PI}=[0.02,0.28]$. As can be seen in Fig. 5b, COVID-19

357 concern rose with social distancing behavior, and then plateaued. Between-state variance in

358 COVID-19 concern levels was larger than zero $\mathrm{SD}=0.05,95 \% \mathrm{PI}=[0.02,0.08]$. A non-linear

359 component is significant also for non-specific anxiety $\mathrm{SD}=0.08,95 \% \mathrm{PI}=[0.003,0.31]$, which

360 also varied considerably between states $\mathrm{SD}=0.06,95 \% \mathrm{PI}=[0.03,0.09]$. 
361 Overall, COVID-19 concern and non-specific anxiety are moderately correlated $\mathrm{r}=0.44, \mathrm{p}<0.001$.

362 Fitting regression models. Cost-benefit rationality and goal-rationality were estimated using a set

363 of multilevel regression models, predicting waiting, satisfaction or memory from wait duration,

364 usefulness judgements, question type, COVID-19 concern and non-specific anxiety. Logistic

365 regression was used for waiting and memory responses and ordered-logistic regression for

366 satisfaction ratings ${ }^{41}$. Models included maximal random effect structure and were fit to the data

367 using Hamiltonian Monte Carlo sampling implemented in the Stan language. Regularizing priors

368 were used to facilitate estimation (Table S1). All predictors were centered and scaled prior to

369 fitting. We report coefficients for duration on the scale of the original data, and for all ratings-

370 based predictors on a standardized scale. Four Monte-Carlo chains were run for each model,

371 collecting 2000 samples each after a 1500 sample warmup period (for the mediation model below

3723000 samples were collected due to model complexity). Convergence was assessed using the $\hat{R}$

373 metric, and visual inspection of trace plots. For all models mentioned in the main text,

374 coefficients for covariates or nuisance parameters are reported in the Supplementary Information.

375 All models in which waiting choices were the dependent variable included usefulness judgments

376 as a predictor. Usefulness was judged on an ordinal Likert scale with only two ratings made by

377 each participant for each question. Thus, we first fit usefulness ratings with a two-parameter

378 ordinal item response theory (IRT) model to extract judged usefulness estimates on a metric

379 scale, rather than apply an averaging operation to the raw ordinal data ${ }^{41,42}$. The model included a

380 term for the average usefulness of each question, and the average rating of each participant. Using

381 R syntax, this is the IRT model fit to usefulness judgments:

382

useful_me, useful_others $\sim(1 \mid$ participant $)+(1 \mid$ question $)$, 
385 We chose an ordered-logistic likelihood function for the usefulness ratings, with a separate set of 386 threshold parameters ${ }^{41}$ for each of the two usefulness items (useful for me / useful for others), to 387 allow for different use of the Likert scale for these two items. The model was fit to usefulness 388 ratings using maximum a posteriori (MAP) estimation with the Stan language ${ }^{43}$. We used the 389 MAP estimates for each question's usefulness in all subsequent models in which usefulness is a 390 predictor. These estimates are highly correlated with the raw averages of the ordinal ratings $391 \mathrm{r}=0.99, \mathrm{p}<0.001$.

392 Assessing cost-benefit rationality. A rational account of curiosity would predict increased waiting

393 for questions with a rise in judged usefulness, and a fall in wait duration. Satisfaction ratings and 394 subsequent memory have been shown to follow curiosity ${ }^{14}$, and so similar predictions can be 395 made for these dependent variables.

396 In order to assess cost-benefit rationality of participants' behavior, we constructed regression 397 models predicting curiosity-driven behavior using judged usefulness, wait duration, the type of 398 question (COVID-19 / general) and all of the interactions between these three factors. We used a 399 multilevel logistic regression to predict waiting and memory from the combination of these 400 predictors, and a multilevel ordered-logistic regression to predict satisfaction ratings. The model 401 was fit with by-participant intercept and slope for all predictors, and by-question intercept and 402 slope for wait duration. Using R syntax, these are the three regression models fit to the data: $403 \quad$ waited $\sim$ wait duration * usefulness * question type + $404+($ wait duration * usefulness * question type $\mid$ participant $)+($ wait duration $\mid$ question $)$, family $=$ bernoulli() Eqn. 2 
satisfaction $\sim$ wait duration * usefulness * question type + + (wait duration * usefulness * question type $\mid$ participant $)+($ wait duration $\mid$ question), family $=$ ordered(link = "logit") Eqn. 3

recalled $\sim$ wait duration $*$ usefulness * question type +

+ (wait duration * usefulness * question type | participant) + (wait duration | question),

414 Assessing goal rationality. We added COVID-19 concern and non-specific anxiety as predictors

415 to a second set of models to assess the effect of motivational state on curiosity driven behavior.

416 Since the first set of models did not reveal any significant interaction with wait-duration in

417 predicting waiting, and no effect of wait duration in predicting memory or satisfaction, these

418 effects were dropped from the second set of models. Using R syntax, these are the three

419 regression models fit to the data:

420 waited $\sim$ wait duration + usefulness * question type * COVID-19 concern + usefulness *

$421 \quad *$ question type * non-specific anxiety +

$422+($ wait duration + usefulness * question type $\mid$ participant $)+$

$423+$ (wait duration + COVID-19 concern + non-specific anxiety $\mid$ question $),$

satisfaction $\sim$ usefulness $*$ question type * COVID-19 concern + usefulness *

* question type * non-specific anxiety + (usefulness * question type $\mid$ participant $)+$ $+($ COVID-19 concern + non-specific anxiety $\mid$ question $)$,

family $=$ ordered(link = "logit")

Eqn. 6 
recalled $\sim$ usefulness * question type * COVID-19 concern + usefulness *

* question type * non-specific anxiety + (usefulness * question type | participant) + $+($ COVID-19 concern + non-specific anxiety | question $)$,

435 Assessing the effect of motivational states on usefulness judgments. The rational framework 436 predicts that motivational effects on curiosity operate by changing the personal utility of 437 questions. Hence, we were interested to see whether mean usefulness judgement levels change 438 with motivational states, operationalized as COVID-19 concern and non-specific anxiety. We fit a 439 multilevel ordered-logistic regression model to usefulness judgements with the goal of estimating 440 the effect of COVID-19 concern and non-specific anxiety on usefulness judgements. Using R 441 syntax, this is the regression model fit to the data: useful_me, useful_others $\sim$ question type * COVID-19 concern + question type * $*$ non-specific anxiety $+(1+$ question type $\mid$ participant $)+$ $+(1+$ COVID-19 concern + non-specific anxiety | question $)$,

446 Here again we fit separate threshold parameters for each usefulness item.

447 Mediation model. As a further test of our rational framework of curiosity, we determined whether

448 the data are congruent with the notion that motivational states influence curiosity by changing 449 personal utility. While the data presented here cannot strictly support a causal account of such 450 sort, joint statistical analysis of waiting choices and usefulness judgments can still validate 451 whether it conforms with the predictions of the rational framework. In order to validate this 
452 prediction, we fit a joint regression model (akin to mediation models) defined by the following

453 two regression equations in R syntax:

useful_me, useful_others $\sim 1+$ question type * COVID-19 concern + $+(1+$ question type $\mid$ participant $)+$

$+(1+$ anxiety $\mid$ question $)$, family $=\operatorname{ordered}(\operatorname{link}=$ "logit" $) \quad$ Eqn. 9

$$
\text { waited } \sim 1+\text { question type * COVID-19 concern }+ \text { usefulness }+
$$$$
+(1+\text { question type }+ \text { usefulness } \mid \text { participant })+
$$

461 The first equation defines the mediator model in mediation analysis parlance, and the second the 462 outcome model. This joint model allowed us to estimate the extent to which both the specific and 463 the generalized effects of COVID-19 concern on waiting are mediated by usefulness judgments.

\section{Acknowledgments}

465 The authors thank Ohad Dan for useful discussion and Nitai Kerem for his help with coding 466 recalled answers. Funding was provided by a Templeton Foundation Science of Virtues grant 467 (\#60844 to DS and RRH). Funders had no role in planning or executing the research reported 468 here.

469 Author Contributions

470 YA, CBM, RRH and DS designed research; YA collected data; YA, CvG and ML analyzed data;

471 YA, RRH and DS wrote the first draft; CBM, CvG and ML edited the paper; RRH and DS

472 supervised.

473 Competing Interest Statement:

474 Authors declare no competing interests. 


\section{References}

476 1. Loewenstein, G. The psychology of curiosity: A review and reinterpretation. Psychol.

477 Bull. 116, 75 (1994).

478 2. Berlyne, D. E. A theory of human curiosity. Br. J. Psychol. 45, 180 (1954).

479 3. Blanchard, T. C., Hayden, B. Y. \& Bromberg-Martin, E. S. Orbitofrontal cortex uses

480 distinct codes for different choice attributes in decisions motivated by curiosity. Neuron

$481 \quad \mathbf{8 5}, 602-614(2015)$.

482 4. Lau, J. K. L., Ozono, H., Kuratomi, K., Komiya, A. \& Murayama, K. Shared striatal 483 activity in decisions to satisfy curiosity and hunger at the risk of electric shocks. Nat.

484 Hum. Behav. 4, 531-543 (2020).

485 5. Hsee, C. K. \& Ruan, B. The Pandora effect: The power and peril of curiosity. Psychol. $486 \quad$ Sci. 27, 659-666(2016).

487 6. Chater, N. \& Loewenstein, G. The under-appreciated drive for sense-making. J. Econ. 488 Behav. Organ. 126, 137-154 (2016).

489 7. Dubey, R. \& Griffiths, T. L. Reconciling Novelty and Complexity Through a Rational $490 \quad$ Analysis of Curiosity. Psychol. Rev. 127, 455-476 (2019).

491 8. Kruglanski, A. W., Jasko, K. \& Friston, K. All Thinking is 'Wishful' Thinking. Trends 492 Cogn. Sci. 24, 413-424 (2020).

493 9. Gruber, M. J., Gelman, B. D. \& Ranganath, C. States of curiosity modulate hippocampus- 
dependent learning via the dopaminergic circuit. Neuron 84, 486-496 (2014).

495 10. Stahl, A. E. \& Feigenson, L. Observing the unexpected enhances infants' learning and 496 exploration. Science (80-. ). 348, 91-94 (2015).

497 11. Kobayashi, K., Ravaioli, S., Baranès, A., Woodford, M. \& Gottlieb, J. Diverse motives for 498 human curiosity. Nat. Hum. Behav. 3, 587-595 (2019).

499 12. Gottlieb, J., Oudeyer, P.-Y., Lopes, M. \& Baranes, A. Information-seeking, curiosity, and 500 attention: computational and neural mechanisms. Trends Cogn. Sci. 17, 585-593 (2013).

501 13. Kang, M. J. et al. The Wick in the Candle of Learning. Psychol. Sci. 20, 963-973 (2009).

502 14. Marvin, C. B. \& Shohamy, D. Curiosity and reward: Valence predicts choice and 503 information prediction errors enhance learning. J. Exp. Psychol. Gen. 145, 266-272 $504 \quad$ (2016).

505 15. Peterson, E. G. Supporting curiosity in schools and classrooms. Curr. Opin. Behav. Sci. $506 \quad 35,7-13(2020)$.

507 16. Schwartenbeck, P. et al. Computational mechanisms of curiosity and goal-directed $508 \quad$ exploration. Elife 8, 1-45 (2019).

509 17. Schulz, E. \& Gershman, S. J. The algorithmic architecture of exploration in the human $510 \quad$ brain. Curr. Opin. Neurobiol. 55, 7-14 (2019).

511 18. Zarocostas, J. How to fight an infodemic. Lancet 395, 676 (2020). 
512 19. Johnson, B. B. \& Slovic, P. Presenting uncertainty in health risk assessment: initial studies 513 of its effects on risk perception and trust. Risk Anal. 15, 485-494 (1995).

514 20. Van Der Bles, A. M., van der Linden, S., Freeman, A. L. J. \& Spiegelhalter, D. J. The 515 effects of communicating uncertainty on public trust in facts and numbers. Proc. Natl. $516 \quad$ Acad. Sci. 117, 7672-7683 (2020).

517 21. Vosoughi, S., Roy, D. \& Aral, S. The spread of true and false news online. Science (80-. ). $518 \quad 359,1146-1151(2018)$.

519 22. Guess, A., Nagler, J. \& Tucker, J. Less than you think: Prevalence and predictors of fake 520 news dissemination on Facebook. Sci. Adv. 5, eaau4586 (2019).

521 23. Norem, J. K. \& Cantor, N. Defensive pessimism: Harnessing anxiety as motivation. $J$. $522 \quad$ Pers. Soc. Psychol. 51, 1208 (1986).

523 24. Eldar, E., Rutledge, R. B., Dolan, R. J. \& Niv, Y. Mood as Representation of Momentum. $524 \quad$ Trends Cogn. Sci. 20, 15-24 (2016).

525 25. Dickinson, A. \& Balleine, B. The role of learning in the operation of motivational 526 systems. Stevens' Handb. Exp. Psychol. (2002).

527 26. Niv, Y., Joel, D. \& Dayan, P. A normative perspective on motivation. Trends Cogn. Sci. $528 \quad \mathbf{1 0}, 375-381(2006)$.

529 27. Förster, J., Liberman, N. \& Friedman, R. S. What do we prime? On distinguishing 530 between semantic priming, procedural priming, and goal priming. Oxford Handb. Hum. 
action 173-192 (2009).

532 28. Dweck, C. S. \& Leggett, E. L. A social-cognitive approach to motivation and personality.

$533 \quad$ Psychol. Rev. 95, 256 (1988).

534 29. Admon, R. \& Pizzagalli, D. A. Dysfunctional reward processing in depression. Curr.

$535 \quad$ Opin. Psychol. 4, 114-118 (2015).

536 30. Rodrigue, J. R., Olson, K. R. \& Markley, R. P. Induced mood and curiosity. Cognit. Ther.

$537 \quad$ Res. 11, 101-106 (1987).

538 31. Anderson, J. R. \& Schooler, L. J. Reflections of the environment in memory. Psychol. Sci.

$539 \quad 2,396-408(1991)$.

$540 \quad 32 . \quad$ Wimber, M., Alink, A., Charest, I., Kriegeskorte, N. \& Anderson, M. C. Retrieval induces 541 adaptive forgetting of competing memories via cortical pattern suppression. Nat. Neurosci.

$542 \quad \mathbf{1 8}, 582-589(2015)$.

543 33. Dugas, M. J., Freeston, M. H. \& Ladouceur, R. Intolerance of uncertainty and problem $544 \quad$ orientation in worry. Cognit. Ther. Res. 21, 593-606 (1997).

545 34. Carleton, R. N. et al. Increasingly certain about uncertainty: Intolerance of uncertainty 546 across anxiety and depression. J. Anxiety Disord. 26, 468-479 (2012).

547 35. Szumowska, E. \& Kruglanski, A. W. Curiosity as end and means. Curr. Opin. Behav. Sci. $548 \quad 35,35-39(2020)$.

549 36. Kobayashi, K. \& Hsu, M. Common neural code for reward and information value. Proc. 
Natl. Acad. Sci. U. S. A. 116, 13061-13066 (2019).

551 37. Cervera, R. L., Wang, M. Z. \& Hayden, B. Y. Systems neuroscience of curiosity. Curr. $552 \quad$ Opin. Behav. Sci. 35, 48-55 (2020).

553 38. Pathak, D., Agrawal, P., Efros, A. A. \& Darrell, T. Curiosity-driven exploration by self554 supervised prediction. in Proceedings of the IEEE Conference on Computer Vision and 555 Pattern Recognition Workshops 16-17 (2017).

556 39. Oba, S. et al. A Bayesian missing value estimation method for gene expression profile 557 data. Bioinformatics 19, 2088-2096 (2003).

558 40. Neuhaus, J. O. \& Wrigley, C. The quartimax method: An analytic approach to orthogonal 559 simple structure 1. Br. J. Stat. Psychol. 7, 81-91 (1954).

560 41. Bürkner, P.-C. \& Vuorre, M. Ordinal regression models in psychology: A tutorial. $A d v$. $561 \quad$ Methods Pract. Psychol. Sci. 2, 77-101 (2019).

562 42. Bürkner, P.-C. Bayesian Item Response Modeling in R with brms and Stan. arXiv Prepr. $563 \quad$ arXiv1905.09501 (2019).

564 43. Gelman, A. et al. Bayesian data analysis. (Chapman and Hall/CRC, 2013).

565 44. Unacast. Unacast Social Distancing Dataset. (2020). Available at: 566 https://www.unacast.com/data-for-good. 